\title{
Utilization of Healthcare Services and Drug Consumption in Fibromyalgia: A Cross-Sectional Analysis of the Clalit Health Service Database
}

\author{
Omer Gendelman ${ }^{1}$, Raz Shapira ${ }^{2}$, Shmuel Tiosano ${ }^{3}$, Yuval Kuntzman ${ }^{4}$, Avishai Tsur ${ }^{4}$, \\ Aliza Hakimian ${ }^{4}$, doron Comaneshter ${ }^{5}$, Arnon Cohen $^{5}$, Dan Buskila ${ }^{6}$, and Howard Amital ${ }^{1}$ \\ ${ }^{1}$ Affiliation not available \\ ${ }^{2}$ Sackler Faculty of Medicine, Tel-Aviv University, Tel-Aviv, Israel \\ ${ }^{3}$ Department of Medicine 'B', Zabludowicz Center for Autoimmune Diseases, Sheba Medical \\ Center, Tel-Hashomer, Israel, Sackler Faculty of Medicine, Tel-Aviv University, Israel \\ ${ }^{4}$ Tel Aviv University Sackler Faculty of Medicine \\ ${ }^{5}$ Clalit Health Services Tel Aviv \\ ${ }^{6}$ Ben-Gurion University of the Negev
}

July 4, 2021

\begin{abstract}
Abstract Aim: To investigate the health care utilization and drug consumption of patients with fibromyalgia (FM). Materials and Methods: This is a cross-sectional study using the Clalit Health Care database. Clalit is the largest HMO in Israel, serving more than 4.4 million enrollees. We identified FM patients and age and sex-matched controls. Indicators of healthcare utilization and drug consumption were extracted and analyzed for both groups. Results: The study included 14,296 FM patients and 71,324 controls. The mean age was 56 years, with a female predominance of $92 \%$. The mean number of visits across of all healthcare services (hospitalizations, emergency department visit, general practitioner clinic visits, rheumatology clinic visits and pain clinic visits) and the mean difference (MD) were significantly higher for FM patients compared to controls (MD 0.66, $\mathrm{p}<0.001$; MD 0.23, $<<0.001$; MD 7.49, $<<0.001$; MD 0.31, p <0.001; MD 0.13, p <0.001), respectively. Drug use was significantly and consistently higher among FM patients compared to controls; NSAIDs (non-steroidal anti-inflammatory drugs) OR 2.56, $\mathrm{P}<0.001$; Opioids OR 4.23, $\mathrm{P}<0.001$; TCA (tricyclic antidepressants) OR 8.21, $\mathrm{P}<0.001$; Gabapentinoids OR 6.31, $\mathrm{P}<0.001$; SSRI (selective serotonin reuptake inhibitors) OR 2.07, $\mathrm{P}<0.001$; SNRI (serotonin-norepinephrine reuptake inhibitor) OR 7.43, $\mathrm{P}<0.001$. Conclusion: Healthcare utilization and drug use are substantially higher among patients with fibromyalgia compared to controls
\end{abstract}

\section{Hosted file}

fm-healthcare_IJCP.docx available at https://authorea.com/users/423694/articles/528973utilization-of-healthcare-services-and-drug-consumption-in-fibromyalgia-a-crosssectional-analysis-of-the-clalit-health-service-database 